OPEN ACCESS

Edited by:

Daniela Tropea,

Trinity College Dublin, Ireland

Reviewed by:

Pawan Gupta

University of Illinois at Chicago, USA

Mihai Moldovan,

University of Copenhagen, Denmark

${ }^{*}$ Correspondence:

Dongsheng Fan

dsfan2010@aliyun.com

Received: 08 September 2016 Accepted: 17 November 2016 Published: 06 December 2016

Citation:

Wu C and Fan D (2016) A Novel Missense Mutation of the DDHD1 Gene Associated with Juvenile Amyotrophic Lateral Sclerosis. Front. Aging Neurosci. 8:291 doi: 10.3389/fnagi.2016.00291

\section{A Novel Missense Mutation of the DDHD1 Gene Associated with Juvenile Amyotrophic Lateral Sclerosis}

\author{
Chujun Wu and Dongsheng Fan* \\ Department of Neurology, Peking University Third Hospital, Beijing, China
}

Background: Juvenile amyotrophic lateral sclerosis (JALS) is a rare form of ALS with an onset age of less than 25 years and is frequently thought to be genetic in origin. DDHD1 gene mutations have been reported to be associated with the SPG28 subtype of autosomal recessive HSP but have never been reported in jALS patients.

Methods: Gene screens for the causative genes of ALS, HSP and CMT using next-generation sequencing (NGS) technologies were performed on a jALS patient. Sanger sequencing was used to validate identified variants and perform segregation analysis.

Results: We identified a novel c.1483A>G (p.Met495Val) homozygous missense mutation of the DDHD1 gene in the jALS patient. All of his parents and young bother were heterozygous for this mutation. The mutation was not found in 800 Chinese control subjects or the database of dbSNP, ExAC and 1000G.

Conclusion: The novel c.1483A>G (p.Met495Val) missense mutation of the DDHD1 gene could be a causative mutation of autosomal recessive jALS.

Keywords: amyotrophic lateral sclerosis, DDHD1 gene, hereditary spastic paraplegia, juvenile, mutation

\section{INTRODUCTION}

Juvenile amyotrophic lateral sclerosis (jALS) is a rare form of ALS with an onset age of less than 25 years and is thought to more frequently have a genetic origin than the adult-onset forms. Juvenile ALS is a clinically and genetically heterogeneous disease. Mutations in ALS2, SETX and SPG11 are known to cause familial jALS with slow disease progression (Orban et al., 2007). In recent literatures, SIGMAR1 have been reported to be a new causative gene of autosomal recessive jALS (Al-Saif et al., 2011; Ullah et al., 2015). However, in sporadic juvenile ALS patients, mutations in Fus are the most frequent genetic cause (Hübers et al., 2015; Zou et al., 2016). On the contrary to slow progression in familial jALS, sporadic jALS patients with FUS or SOD1 mutations experienced aggressive progression and short survival times (Zou et al., 2016). Some of jALS causative genes have also been reported in other diseases, such as ALS2 and SPG11 causing hereditary spastic paraplegia (HSP) as well (Eymard-Pierre et al., 2002; Klebe et al., 2015). In this report, we describe a jALS patient with a novel missense mutation in the DDHD1 gene, which is a member of the intracellular phospholipase A1 gene family and involved in the regulation of mitochondrial function. Associations have been reported between mutations in the DDHD1 gene and the SPG28 subtype of autosomal recessive HSP but have never been reported in jALS patients. 


\section{MATERIALS AND METHODS}

\section{Subjects}

The pedigree for the family is presented in the Figure 1A. The clinical characteristics of the patient will be discussed in results section.

\section{Ethics Statement}

The institutional ethics committee of Peking University Third Hospital approved this study (IRB00006761). Written, informed consent was obtained from each participant.

\section{Genetic Analysis}

We obtained blood sample from affected and unaffected subjects in the pedigree (III-7, III-8, IV-1, IV-2). Next generation sequencing (NGS)was performed on an Illumina GAIIx platform to screen for variations in the patient, which covers the coding exons and flanking intronic sequences of 190 causative genes of ALS, HSP, and Charcot-Marie-Tooth disease (CMT) (Supplementary file). Identified variants in NGS were validated by Sanger sequencing. In order to perform segregation analysis, all of the patients' parents and young brother were screened for the identified variants using Sanger sequencing.

\section{RESULTS}

\section{Clinical Features}

The patient (IV-1) was a 24-year-old male who developed walking difficulties due to leg weakness beginning at 16 years of age and exhibited atrophy of the bilateral first interosseous

\section{A Family pedigree}

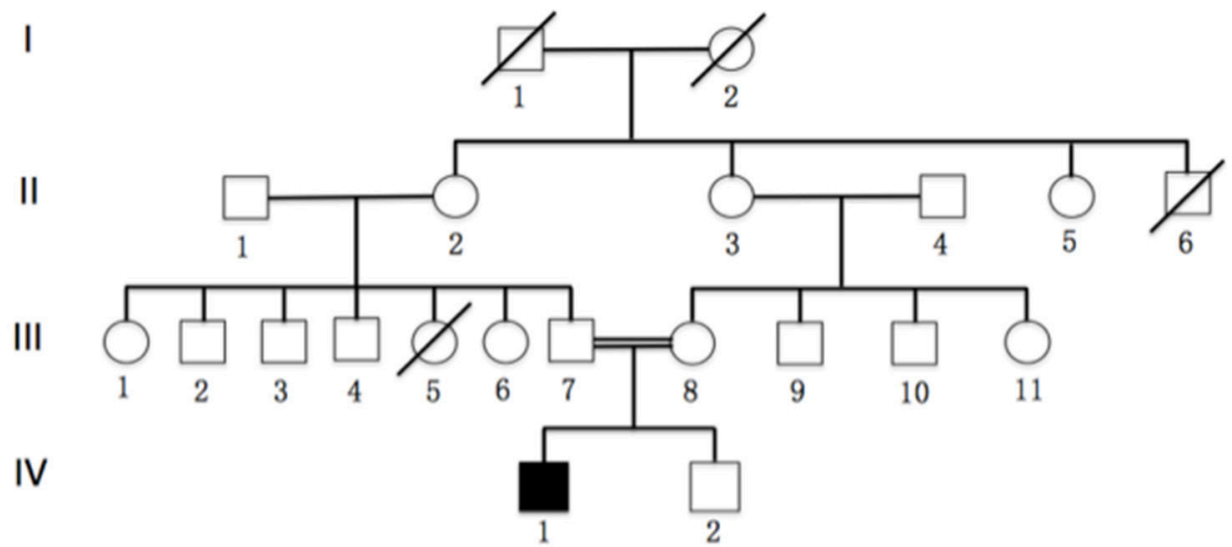

B Genomic sequence electropherograms
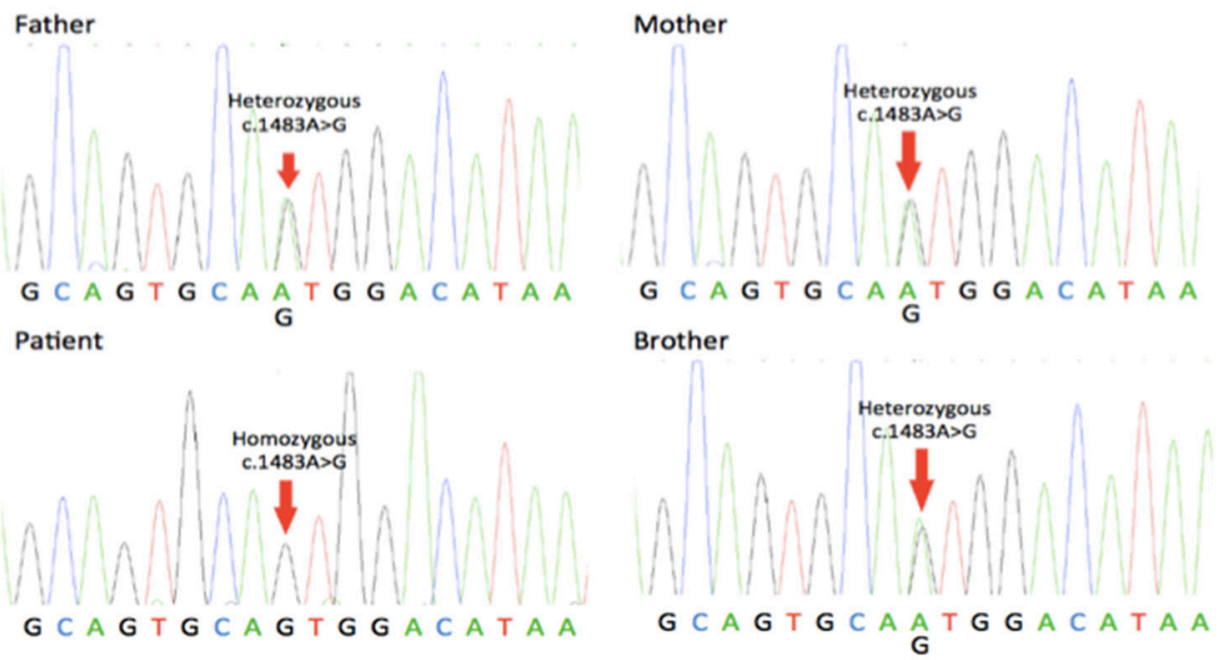

FIGURE 1 | Family pedigree and genomic sequence electropherograms. (A) Family pedigree. The jALS patient was born to consanguineous Chinese parents. All of his parents and young brother were healthy. Males and Females are represented as squares and circles, respectively. The filled symbol represents the affected patient while unaffected individuals are represented by clear symbols. Crossed circles or squares represent deceased individuals. A double line indicates consanguineous mating. (B) Genomic sequence electropherograms. The jALS patient carried a novel homozygous c.1483A>G (p.Met495Val) missense mutation of the $D D H D 1$ gene, which was not detected in 800 healthy unrelated Chinese individuals. All of his parents and young brother were heterozygous for this mutation. Pedigree analysis suggested that the disease is consistent with autosomal recessive inheritance. 
muscles accompanied by a mild weakness in the hands 1 year later. These symptoms have progressed slowly. Besides, he didn't complain of any sensory abnormality. He was born to consanguineous Chinese parents. His parents and younger brother were clinically healthy. All of them didn't present any weakness or atrophy of muscle.

Neurologic examinations were performed for the patient (IV1), his parents (III-7, III-8) and young brother (IV-2). In the patient, examinations revealed a steppage gait, atrophy of the bilateral interosseous and thenar muscles with a split-hand sign, mild weakness in the hands and lower limbs, hyperreflexia in all limbs with positive bilateral Babinski signs and Hoffmann signs, and disappearance of abdominal reflexes. Sensation and coordination were normal. As for the patients' parents and young brother, neurological examinations showed no muscle atrophy, normal tendon reflex and negative pathological reflex.

Needle EMG was performed on the patient. The results showed neurogenic changes, including fibrillation potentials and positive sharp waves, in four regions (brainstem, cervical, thoracic, and lumbosacral spinal cord). Nerve conduction studies revealed decreases in the amplitude of compound motor and sensory action potentials with an almost normal nerve conduction velocity. The structure brain and cervical MRIs were normal. Upon ${ }^{1} \mathrm{H}$-MRS examination, no pathological lactate accumulation was found in the cerebrospinal fluid of lateral ventricles.

\section{Genetic Results}

Gene screens for the causative genes of ALS, HSP, and CMT using NGS technologies identified a novel homozygous c.1483A>G (p.Met495Val)(RefSeq NM_001160147.1) missense mutation of the DDHD1 gene in the patient (Figure 1B), which was verified by Sanger sequencing. Meanwhile, Sanger sequencing revealed all of his parents and young brother were heterozygous for this mutation (Figure 1B). The mutation was not detected in 800 healthy unrelated Chinese individuals by whole exome sequencing or NGS.

\section{DISCUSSION}

DDHD1, also known as SPG28 or PA-PLA1, is a member of the intracellular phospholipase A1 gene family. The protein encoded by the DDHD1 gene is a cytosolic protein with some mitochondrial localization and is involved in the regulation of mitochondrial dynamics via phosphatidic acid (PA) (Baba et al., 2014). The PA on the surface of mitochondria is known to regulate mitochondrial fusion. DDHD1 is the first identified intracellular phospholipase A1 and preferentially hydrolyzes $\mathrm{PA}$ in vitro. The DDHD1 pathogenic mutations cause reduced PA-PLA1 activity, and the resultant increased PA content on the surface of mitochondria might cause the impairment of mitochondrial fusion and lead to the dysfunction of mitochondria (Tesson et al., 2012). The previous study has showed the ectopic expression of DDHD1 in HeLa cells induced mitochondrial fragmentation, whereas its depletion caused mitochondrial elongation. Gene disruption of DDHD1 in mice caused sperm malformation due to mitochondrial organization defects (Baba et al., 2014). In patients harboring pathogenic DDHD1 gene mutations, histochemical analyses in muscle showed mitochondrial alterations, and multiple mitochondrial DNA (mtDNA) deletions were evident (Mignarri et al., 2016). Besides, mitochondrial respiration rate, total cellular and mitochondrial ATP content were found to be significantly lower in lymphoblast from SPG28 patients (Tesson et al., 2012). In brain ${ }^{1} \mathrm{H}$-MRS analysis, a mild pathological accumulation of lactate in the cerebrospinal fluid was detected in a SPG28 patient with 20 years of disease duration, (Liguori et al., 2014) but not other three patients with shorter disease duration.(Liguori et al., 2014; Mignarri et al., 2016)

In previous studies, 8 mutations of the DDHD1 gene have been reported in autosomal recessive HSP patients, including three nonsense mutations, three frame-shift mutations and two mutations affecting the mRNA splicing site (Tesson et al., 2012; Liguori et al., 2014; Mignarri et al., 2016; Miura et al., 2016). All of these mutations predict changes in the protein translation of the DDHD domain, which is responsible for the phospholipase activity of the DDHD1 protein, thereby leading to a loss of function in the protein and finally mitochondrial dysfunction.

In our report, the patient carried a novel homozygous c.1483A $>$ G (p.Met495Val) missense mutation located in exon 7 of the DDHD1 gene (Figure 2). His parents and younger brother were heterozygous for this mutation. Pedigree analysis suggested that the disease is consistent with autosomal recessive inheritance. The mutation was not detected in 800 healthy unrelated Chinese individuals, suggesting that the mutation is not present in the normal Chinese population.

Furthermore, this new mutation, located in a conserved domain (Figure 3), was not found in dbSNP, ExAC or $1000 \mathrm{G}$ database and was predicted to be disease causing by MutationTaster, possibly damaging by Polyphen-2 and affecting protein function by SIFT. So we concluded this variant was a pathogenic mutation rather than a polymorphism.

Mitochondrial dysfunction is one of the important pathophysiological mechanisms in ALS (Mancuso and Navarro, 2015). In our report, the novel homozygous missense DDHD1 mutation might cause jALS via mitochondrial dysfunction. Unlike the previously reported mutations, the new mutation may not change the protein primary structure but rather the three-dimensional structure of the DDHD domain, ultimately causing a loss of function in the protein. However, further functional studies are needed to confirm mitochondrial dysfunction in ALS patients with DDHD1 gene mutations.

Patients with DDHD1 gene mutations have presented pure or mildly complicated HSP in previous studies (Bouslam et al., 2005; Tesson et al., 2012; Liguori et al., 2014; Mignarri et al., 2016; Miura et al., 2016). Tongue fasciculation with wasting have been reported previously, (Liguori et al., 2014) indicating that DDHD1 gene mutations may influence lower motor neuron (LMN) function. In this patient, muscle atrophy of hands with a splithand sign was apparent, and needle EMG confirmed neurogenic damage in four regions. We believe that the decreased amplitude of compound motor action potentials was the result of severe LMN damage. Apart from motor system involvement, subclinical sensory axonal neuropathy was detected in this patient. In previous reports, clinical and subclinical sensory defects had been reported in HSP patients with DDHD1 gene mutations, 


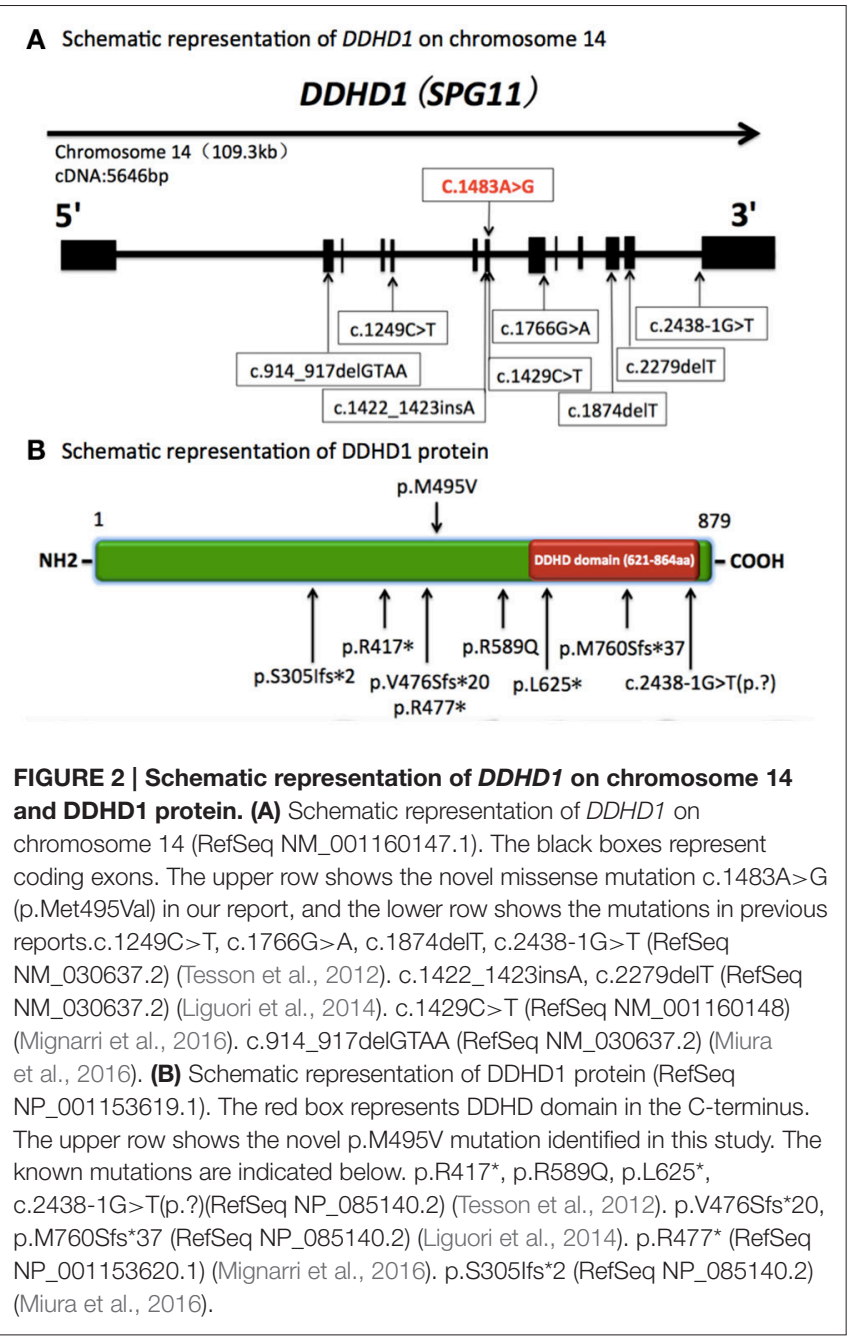

which reflected DDHD1 gene mutations might cause sensory defects (Bouslam et al., 2005; Liguori et al., 2014). Moreover, subclinical sensory abnormalities, peripheral as well as central levels, could be found in ALS patients who didn't have other known potential causes of polyneuropathy, such as diabetes mellitus (Pugdahl et al., 2007; Iglesias et al., 2015; Isak et al., 2016). A multicenter study reported $22.7 \%$ patients with ALS had sensory nerve action potentials (SNAPs) abnormalities in at least one nerve (Pugdahl et al., 2007). Although pathophysiological mechanisms underlying sensory abnormalities remains to be further clarified, these findings reflected that ALS might be a multi-systemic disorder involving other systems than motor. In the revision of the EI Escorial criteria 2015, sensory impairment does not exclude ALS diagnosis. Besides, UMN and LMN impairment, rather than subclinical sensory impairment, was the dominant presentation in this patient. Finally, the patient was diagnosed with lab-supported probable jALS. Considering the clinical history of this patient and previously reported cases, we believe that jALS patients with DDHD1 mutations could have slow disease progression, similar to that of other familial jALS patients.

\begin{tabular}{|c|c|c|}
\hline & \multicolumn{2}{|c|}{ p.Met495 } \\
\hline Homo sapiens & $D M L N S S A$ & $M D I M Y Y T S$ \\
\hline Pan troglodytes & $D M L N S S A$ & $M D I M Y Y T S$ \\
\hline Macaca mulatta & $D M L N S S A$ & $M D I M Y Y T S$ \\
\hline Oryctolagus cuniculus & $D M L N S S A$ & $M D I M Y Y T S$ \\
\hline Rattus norvegicus & $D M L N S S A$ & $M D I M Y Y T S$ \\
\hline Mus musculus & $D M L N S S A$ & $M D I M Y Y T S$ \\
\hline Gallus gallus & $D M L N S S A$ & $M D I M Y Y T S$ \\
\hline Xenopus tropicalis & $D M L N S S A$ & M D I M Y Y T S \\
\hline Danio rerio & $D M L N S S A$ & $M D I M Y Y T S$ \\
\hline Felis catus & $D M L N S S A$ & $M D I M Y Y T S$ \\
\hline Canis lupus familiaris & D $M$ M L N S S S A & M D I I M Y Y Y T T \\
\hline Bos taurus & D M L N S S & M D I M Y Y T S \\
\hline
\end{tabular}

FIGURE 3 | Conservation of the amino acid affected by the missense mutation. Amino acid sequence alignments around the single amino acid affected by the novel DDHD1 gene mutation are shown for selected species. The amino acid affected by the novel p.Met495Val missense mutation is highlighted by a red rectangle. This novel mutation was located in a very conserved domain, and was predicted to be disease causing by MutationTaster, possibly damaging by Polyphen-2 and affecting protein function by SIFT.

In summary, this is the first case report about the relationship between $D D H D 1$ gene mutations and jALS. Besides of dominant motor system involvement, subclinical sensory impairment was detected in this jALS patient. All of these findings reflected the clinically and genetically heterogeneity of jALS and further proved the genetic overlap between HSP and jALS. Meanwhile, further functional studies are needed to confirm the effects of DDHD1 gene mutations in ALS pathogenesis.

\section{CONCLUSION}

Juvenile ALS is a clinically and genetically heterogeneous disease. As described in our report, we discovered a novel c.1483A $>\mathrm{G}$ (p.Met495Val) missense mutation of the DDHD1 gene that could be a causative mutation of autosomal recessive jALS.

\section{AUTHOR CONTRIBUTIONS}

DF conceived this study and provided financial support; CW and DF performed the experiments, analyzed the data, and wrote the manuscript.

\section{FUNDING}

This study was supported by the National Natural Science Foundation of China (81030019).

\section{SUPPLEMENTARY MATERIAL}

The Supplementary Material for this article can be found online at: http://journal.frontiersin.org/article/10.3389/fnagi. 2016.00291/full\#supplementary-material 


\section{REFERENCES}

Al-Saif, A., Al-Mohanna, F., and Bohlega, S. (2011). A mutation in sigma1 receptor causes juvenile amyotrophic lateral sclerosis. Ann. Neurol. 70, 913-919. doi: 10.1002/ana.22534

Baba, T., Kashiwagi, Y., Arimitsu, N., Kogure, T., Edo, A., Maruyama, T., et al. (2014). Phosphatidic acid (PA)-preferring phospholipase A1 regulates mitochondrial dynamics. J. Biol. Chem. 289, 11497-11511. doi: 10.1074/jbc. M113.531921

Bouslam, N., Benomar, A., Azzedine, H., Bouhouche, A., Namekawa, M., Klebe, S., et al. (2005). Mapping of a new form of pure autosomal recessive spastic paraplegia (SPG28). Ann. Neurol. 57, 567-571. doi: 10.1002/ana.20416

Eymard-Pierre, E., Lesca, G., Dollet, S., Santorelli, F. M., di Capua, M., Bertini, E., et al. (2002). Infantile-onset ascending hereditary spastic paralysis is associated with mutations in the alsin gene. Am. J. Hum. Genet. 71, 518-527. doi: 10.1086/ 342359

Hübers, A., Just, W., Rosenbohm, A., Müller, K., Marroquin, N., Goebel, I., et al. (2015). De novo FUS mutations are the most frequent genetic cause in earlyonset German ALS patients. Neurobiol. Aging 36, 3117.e1-3117.e6. doi: 10. 1016/j.neurobiolaging.2015.08.005

Iglesias, C., Sangari, S., El Mendili, M. M., Benali, H., Marchand-Pauvert, V., and Pradat, P. F. (2015). Electrophysiological and spinal imaging evidences for sensory dysfunction in amyotrophic lateral sclerosis. BMJ Open 5:e007659. doi: 10.1136/bmjopen-2015-007659

Isak, B., Tankisi, H., Johnsen, B., Pugdahl, K., Finnerup, N. B., and FuglsangFrederiksen, A. (2016). Laser and somatosensory evoked potentials in amyotrophic lateral sclerosis. Clin. Neurophysiol. 127, 3322-3328. doi: 10.1016/ j.clinph.2016.08.008

Klebe, S., Stevanin, G., and Depienne, C. (2015). Clinical and genetic heterogeneity in hereditary spastic paraplegias: from SPG1 to SPG72 and still counting. Rev. Neurol. (Paris).171, 505-530. doi: 10.1016/j.neurol.2015.02.017

Liguori, R., Giannoccaro, M. P., Arnoldi, A., Citterio, A., Tonon, C., Lodi, R., et al. (2014). Impairment of brain and muscle energy metabolism detected by magnetic resonance spectroscopy in hereditary spastic paraparesis type 28 patients with DDHD1 mutations. J. Neurol. 261, 1789-1793. doi: 10.1007/ s00415-014-7418-4

Mancuso, R., and Navarro, X. (2015). Amyotrophic lateral sclerosis: current perspectives from basic research to the clinic. Prog. Neurobiol. 133, 1-26. doi: 10.1016/j.pneurobio.2015.07.004
Mignarri, A., Rubegni, A., Tessa, A., Stefanucci, S., Malandrini, A., Cardaioli, E., et al. (2016). Mitochondrial dysfunction in hereditary spastic paraparesis with mutations in DDHD1/SPG28. J. Neurol. Sci. 362, 287-291. doi: 10.1016/j.jns. 2016.02.007

Miura, S., Morikawa, T., Fujioka, R., Kosaka, K., Yamada, K., Hattori, G., et al. (2016). A novel frameshift mutation of DDHD1 in a Japanese patient with autosomal recessive spastic paraplegia. Eur. J. Med. Genet. 59, 413-416. doi: 10. 1016/j.ejmg.2016.05.010

Orban, P., Devon, R. S., Hayden, M. R., and Leavitt, B. R. (2007). Chapter 15 Juvenile amyotrophic lateral sclerosis. Handb. Clin. Neurol. 82, 301-312. doi: 10.1016/S0072-9752(07)80018-2

Pugdahl, K., Fuglsang-Frederiksen, A., de Carvalho, M., Johnsen, B., Fawcett, P. R., Labarre-Vila, A., et al. (2007). Generalised sensory system abnormalities in amyotrophic lateral sclerosis: a European multicentre study. J. Neurol. Neurosurg. Psychiatr. 78, 746-749. doi: 10.1136/jnnp.2006.098533

Tesson, C., Nawara, M., Salih, M. A. M., Rossignol, R., Zaki, M. S., Al Balwi, M., et al. (2012). Alteration of fatty-acid-metabolizing enzymes affects mitochondrial form and function in hereditary spastic paraplegia. Am. J. Hum. Genet. 91, 1051-1064. doi: 10.1016/j.ajhg.2012.11.001

Ullah, M. I., Ahmad, A., Raza, S. I., Amar, A., Ali, A., Bhatti, A., et al. (2015). In silico analysis of SIGMAR1 variant (rs4879809) segregating in a consanguineous Pakistani family showing amyotrophic lateral sclerosis without frontotemporal lobar dementia. Neurogenetics 16, 299-306. doi: 10.1007/ s10048-015-0453-1

Zou, Z. Y., Liu, M. S., Li, X. G., and Cui, L. Y. (2016). Mutations in FUS are the most frequent genetic cause in juvenile sporadic ALS patients of Chinese origin. Amyotroph. Lateral Scler. Frontotemporal Degener. 17, 249-252. doi: 10.3109/ 21678421.2016.1143012

Conflict of Interest Statement: The authors declare that the research was conducted in the absence of any commercial or financial relationships that could be construed as a potential conflict of interest.

Copyright (c) $2016 \mathrm{Wu}$ and Fan. This is an open-access article distributed under the terms of the Creative Commons Attribution License (CC BY). The use, distribution or reproduction in other forums is permitted, provided the original author(s) or licensor are credited and that the original publication in this journal is cited, in accordance with accepted academic practice. No use, distribution or reproduction is permitted which does not comply with these terms. 